\title{
Proposed Additions to the NCCN Guidelines for Adult Medulloblastoma
}

\author{
Marta Penas-Prado, MD¹; Terri S. Armstrong, PhD, ANP-BC ${ }^{1}$; and Mark R. Gilbert, MD ${ }^{1}$
}

\begin{abstract}
Medulloblastoma is a rare brain tumor that occurs in both children and adults, with patients aged 15 to 39 years accounting for $30 \%$ of all cases. In adults, guidelines for diagnosis and treatment are often based on retrospective data and extrapolated from the pediatric experience due to limited availability of prospective trials or registries involving adults. Importantly, adult patients differ from pediatric patients in many aspects, including the molecular features of the tumor and tolerance to treatment. In 2017, the $\mathrm{NCl}$ was granted support from the Cancer Moonshot initiative to address the challenges and unmet needs of adults with rare central nervous system (CNS) tumors through the $\mathrm{NCl}$ Comprehensive Oncology Network for Evaluating Rare CNS Tumors (NCl-CONNECT). On November 25, 2019, NCl-CONNECT convened a multidisciplinary workshop on adult medulloblastoma. Working groups identified unmet needs in clinical care and research and developed specific action items, including a proposal for inclusion of new items in the NCCN Guidelines for Adult Medulloblastoma, delineated in this review along with the evidence supporting their incorporation. Recommendations included facilitating referral of patients to centers of excellence; promoting patient participation in clinical trials or registries; encouraging use of DNA methylation for confirmation of diagnosis and subgrouping; offering counseling on contraception and fertility preservation; evaluating patients for symptoms and medical management of endocrine, vision, hearing, and neurocognitive deficits; providing psychosocial support and referral to neurorehabilitation; minimizing delays in therapy; and incorporating imaging standards and criteria for progression.
\end{abstract}

J Natl Compr Canc Netw 2020;18(11):1579-1584 doi: $10.6004 /$ jnccn.2020.7650

\footnotetext{
${ }^{1}$ Neuro-Oncology Branch, Center for Cancer Research, NCl, NIH, Bethesda, Maryland.
}

The NCCN Clinical Practice Guidelines in Oncology (NCCN Guidelines) are a wide-ranging set of recommendations detailing sequential management decisions and interventions for patients with cancer, with the goal of improving patient care and outcomes. The NCCN Guidelines provide continuously updated and revised recommendations based on the best available evidence and are the recognized standard for clinical policy in cancer care. ${ }^{1}$ For patients with rare central nervous system (CNS) tumors, such as medulloblastoma in adults, high-quality evidencebased recommendations are rarely available due to limited prospective and randomized data, leading to wide heterogeneity in management. ${ }^{2}$ The current NCCN Guidelines for Adult Medulloblastoma are available online (see pages AMED-1, AMED-2, and AMED-3 in the NCCN Guidelines for CNS Cancers at NCCN.org).

The mission of the NCI Comprehensive Oncology Network for Evaluating Rare CNS Tumors (NCICONNECT), housed in the Neuro-Oncology Branch of the NCI's Center for Cancer Research and supported by the Cancer Moonshot, is to advance understanding of adults with rare CNS tumors by establishing partnerships among patients, advocacy groups, and providers to improve care and treatment (https://www.cancer.gov/ rare-brain-spine-tumor/tumors).$^{3-5}$ One of the mechanisms used to reach this goal is hosting transdisciplinary workshops. ${ }^{3,4}$ On November 25, 2019, NCI-CONNECT convened a workshop on adult medulloblastoma. The proceedings of this workshop are discussed in detail elsewhere. ${ }^{6}$ This was a hands-on multidisciplinary workshop in which clinicians, scientists, and advocacy partners met to discuss areas requiring further development, including understanding patients' needs, deciphering the molecular pathology of adult medulloblastoma, developing suitable preclinical models, optimizing clinical trial design and efficacy endpoints, and launching collaborative efforts in clinical and translational research. Attendees developed an action plan to address challenges and identified an immediate need to update the NCCN Guidelines as one of the early priorities. 


\section{Proposed Additions to the NCCN Guidelines for Adult Medulloblastoma}

This review summarizes a proposal to include 6 new items in the NCCN Guidelines for Adult Medulloblastoma (Table 1) and reviews the literature supporting their inclusion. For topics lacking sufficient published data, recommendations were based on experience and the consensus of workshop attendees.

\section{Facilitate Referral to Specialized Centers or "Centers of Excellence"}

Only approximately 140 new cases of medulloblastoma are diagnosed each year in the United States in patients aged $\geq 15$ years. Patients aged $\geq 40$ years represent $<30$ new cases per year, or $<8 \%$ of all medulloblastoma cases. The estimated prevalence of medulloblastoma in the United States is 1,423 pediatric cases, 1,862 cases of late adolescent and young adult (AYA) cases aged 15 to 39 years (most of whom were likely diagnosed in childhood), 492 cases aged 40 to 64 years, and 58 cases aged $\geq 65$ years. $^{7}$ The lower incidence and prevalence of cases beyond childhood make finding and building expertise to treat adult medulloblastoma difficult, because even academic and brain tumor referral centers only see a small number of such patients each year. ${ }^{8}$

Medulloblastoma is classified as a malignant grade 4 primary CNS tumor by the WHO. It displays rapid growth and the tendency to disseminate within the CNS and systemically, posing many diagnostic and therapeutic challenges across the entire age spectrum. These challenges include the need for prompt and complex multidisciplinary treatment decisions and planning involving surgery (resection and/or cerebrospinal fluid diversion procedures), craniospinal irradiation (CSI), and chemotherapy ${ }^{9,10}$; accurate assessment of imaging findings and neuropathologic diagnosis (including advanced molecular testing for tumor subgrouping and discovery of potential actionable targets); and specialized support for acute, subacute, and late adverse effects and complications derived from tumor and therapy. In addition, adults with medulloblastoma have very limited access to experimental therapies. ${ }^{11}$
The current NCCN Guidelines recommend consideration of "a multidisciplinary review in treatment planning, before surgery and once pathology is available" and "strongly recommend referring patient[s] to a brain tumor center to be evaluated for possible further, more complete surgical resection." ${ }^{\prime}$ Taking this guidance a step further, one of the main workshop recommendations was that adults with medulloblastoma should be referred to specialized centers or "centers of excellence" with expertise in diagnosis and treatment of these patients at any time when critical decisions about their care must be made, both at the time of new diagnosis or at recurrence. This was also recommended in the 2019 European Association of Neuro-Oncology and European Rare Cancer clinical practice guideline for diagnosis, treatment, and follow-up of postpubertal and adult patients with medulloblastoma. ${ }^{10}$

Importantly, there are no official criteria for defining "centers of excellence" in neuro-oncology, and there are no prescribed standards or associated certifications. Centers of excellence in healthcare have previously been described as "specialized programs... which supply exceptionally high concentrations of expertise and related resources centered on particular medical areas and delivered in a comprehensive, interdisciplinary fashion...to afford the best patient outcomes possible." ${ }^{12}$ With only a few exceptions (eg, bariatric surgery centers), hospitals typically self-designate services as "centers of excellence" with varying degrees of rigor of standards.

Referring patients to centers with experience is expected to reduce the likelihood of misdiagnosis and complications that may result from treatment, and potentially improve outcomes, as seen in other more common primary brain tumors. For example, in glioblastoma, the most frequent malignant primary brain tumor in adults, a recent review of the National Cancer Database explored the association of patient outcomes with the type of facility (academic vs nonacademic center) and facility volume (high vs low volume), including data on $>40,000$ patients seen over a decade. ${ }^{13}$ Patients treated at academic centers and high-volume facilities experienced the longest survival rates and had more frequent access to tumor resection, radiotherapy,

\section{Table 1. Proposal for Inclusion of 6 New Items in NCCN Guidelines for Adult Medulloblastoma}

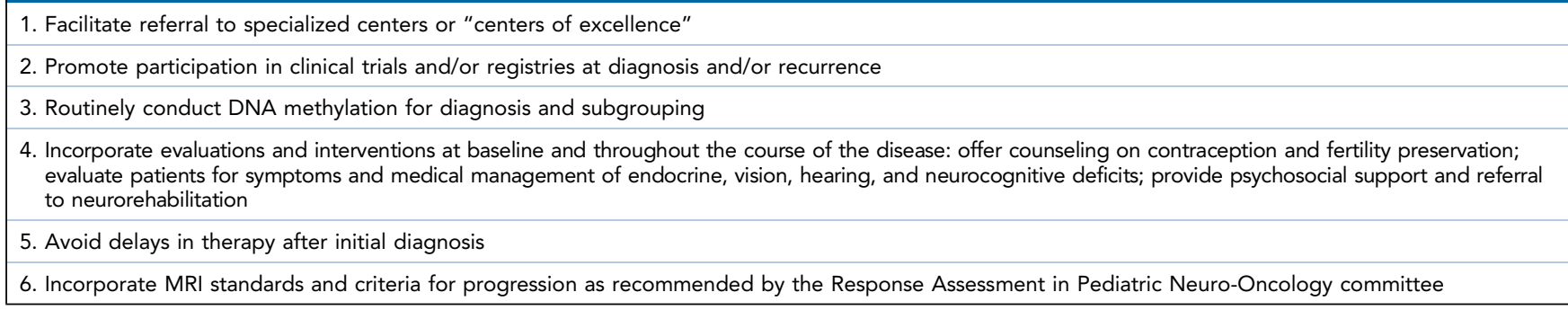


and chemotherapy. Moreover, prolonged inpatient stays, 30-day readmission, and 90-day mortality were significantly decreased at this type of center (by $20 \%$, $22 \%$, and $16 \%$, respectively).

A nationwide cancer registry retrospective study in Finland including $>2,000$ patients with glioblastoma also found improved survival at high-volume centers, ${ }^{14}$ suggesting this may be a consistent finding across different healthcare systems. There is also an increasing body of evidence suggesting lower morbidity and mortality rates in patients undergoing low-grade glioma and glioblastoma surgery by specialized surgeons in high-volume centers, ${ }^{15,16}$ and also suggesting improved survival of patients with glioblastoma receiving chemoradiation in high-volume facilities. ${ }^{17}$ Even though, to the best of our knowledge, data specific to outcomes of adults with medulloblastoma based on the type of facility providing treatment have not been published, it is likely that similar findings would be observed, because medulloblastoma is rarer than glioblastoma, and its management is complex.

Based on the available literature in glioblastoma and other gliomas, one can extrapolate that the basic elements of a center of excellence (those associated with improved outcomes) are, at a minimum, academic high-volume centers with access to specialized multidisciplinary care (neurosurgery, neuropathology, radiation oncology, and neuro-oncology). Other important components particularly relevant for adults with medulloblastoma include availability of advanced molecular testing, regular tumor boards, access to proton radiation therapy, a clinical trial program, patient education seminars and support groups, and social and supportive care services.

An important consideration is that patients with limited resources or living in remote areas might not have access to such centers of excellence. Potential solutions include referrals for expert consultation with care provided in collaboration with local providers, in person, or via telehealth medicine, ${ }^{18,19}$ and the implementation of web-based tumor boards ${ }^{20,21}$ to review such cases and provide multidisciplinary expert advice. One important component of achieving the goal of referring adults with medulloblastoma to centers of excellence is to educate patients, caregivers, healthcare providers, and thirdparty payers about the benefits of being seen at centers with expertise.

\section{Promote Participation in Clinical Trials and/or \\ Registries at Diagnosis and/or Recurrence}

Participation in clinical trials is "especially encouraged" by all NCCN Guidelines for treatment of cancer, and each guideline includes the opening statement "NCCN believes that the best management for any patient with cancer is in a clinical trial," which is also included as a comment at the foot of each algorithm. In addition, specific CNS tumor types, such as anaplastic astrocytoma and ependymoma, mention participation in clinical trials as part of the algorithm itself; however, such a recommendation is not included within the algorithm for adult medulloblastoma. One of the results of the workshop was to further strengthen the recommendation to include adults with medulloblastoma in clinical trials, when available, and in registries or natural history studies, because information from every patient carries great value due to the small number of patients.

Although long-term survival is possible in adults with medulloblastoma, many questions remain regarding optimal upfront therapy. Furthermore, recurrences are typically difficult to treat and often incurable; chronic sequelae of the disease in survivors are understudied; and patients need symptomatic interventions. Data are needed on symptom burden, quality of life, neurocognitive outcomes, and social outcomes, including financial burden. Participation of adults with medulloblastoma in clinical trials is critically needed to address these gaps in knowledge and ultimately improve patient outcomes. Although the design and implementation of clinical trials for patients with rare tumors pose many challenges, ${ }^{11}$ extensive collaborative efforts are key. As an example, 2 large multicenter randomized trials are currently in development by the EORTC and Alliance. These trials are specifically designed for AYAs and adults with newly diagnosed standard-risk medulloblastoma and are primarily evaluating the role of smoothened inhibitors when given along with upfront therapy (CSI and chemotherapy) for newly diagnosed sonic hedgehog subgroup medulloblastoma, but they also include all other medulloblastoma clinical and molecular groups in exploratory arms. There are also ongoing trials enrolling adults with recurrent medulloblastoma (ClinicalTrials.gov identifiers: NCT03173950, NCT03434262, NCT03904862), and a call has been made to liberalize eligibility criteria in future phase I trials to allow participation of patients with rare CNS tumors. ${ }^{22}$

Despite these ongoing clinical trial efforts, adults with both newly diagnosed and recurrent medulloblastoma continue to have limited access to clinical trials. Thus, it is critical to collect patient data in centralized registries or natural history studies that allow remote participation, so that valuable clinical and molecular information from as many patients as possible can increase knowledge about the disease. Examples of these types of studies include the HIT-2000 multicenter prospective observational adult cohort ${ }^{23,24}$ and the ongoing Natural History of and Specimen Banking for People With Tumors of the Central Nervous System (ClinicalTrials.gov identifier: NCT02851706) and Rare CNS 
Tumors Outcomes \& Risk studies (NCT03251989) at NCI, which allow the collection of comprehensive, structured clinical information and the banking of tumor tissue and other specimens.

\section{Routinely Conduct DNA Methylation for}

Diagnosis and Subgrouping

Current NCCN Guidelines for Adult Medulloblastoma ${ }^{1}$ recommend "molecular profiling to identify clinically relevant subtypes...to encourage opportunities for clinical trial involvement." The Principles of Brain Tumor Pathology section (see page BRAIN-F in the NCCN Guidelines for CNS Cancers, available at NCCN.org) recommends that " $[\mathrm{m}]$ edulloblastoma testing should be referred to academic tertiary centers with expertise in this area," and differentiating among the current subgroups described in the WHO Classification of Tumors of the Central Nervous System ${ }^{25}$ "is best classified by expression arrays, DNA methylation arrays, or an immunohistochemistry [IHC] panel composed of $\beta$-catenin, GAB1, and YAP1." ${ }^{1}$ Gene sequencing is also recommended in sonic hedgehog subgroup medulloblastoma to detect TP53 mutations, given the variety of hotspots in the gene and the prognostic importance of this finding. Finally, it is also clarified that "the diagnosis of medulloblastoma is still made on the basis of light microscopy" because none of the molecular markers associated with each subgroup is unique to medulloblastoma. ${ }^{1}$

Currently, there is no universally accepted gold standard method for subgroup assignment in medulloblastoma, and, as mentioned in the guideline, different methods are used across different institutions. This variability in subgrouping (and in many cases the lack of testing for subgrouping) is an important challenge that makes collaboration difficult and slows research and advances in the field. Genome-wide DNA methylation with application of the brain tumor classifier ${ }^{26}$ along with next-generation sequencing is a reliable and reproducible method to confirm the diagnosis of medulloblastoma and perform subgroup classification; it was supported by workshop attendees as the gold standard for medulloblastoma subgrouping. DNA methylation is already performed for most medulloblastoma cases in some European countries (France, Germany, and the United Kingdom), and it is therefore incorporated in the upcoming EORTC trial for newly diagnosed adult medulloblastoma as part of the central neuropathology review to be performed before initiation of therapy. DNA methylation is also increasingly performed at academic centers in the United States and has important advantages, such as helping reduce diagnostic error, allowing differential diagnosis with other posterior fossa tumors, and distinguishing between group 3 and group 4 tumors, which is not yet possible by readily available IHC markers. Importantly, NCI has observed a change of diagnosis from that rendered by classic histopathologic methods in $11 \%$ of patients with primary CNS tumors by using integrated analysis that incorporates histology, next-generation sequencing, and DNA methylation, similar to that found in a study evaluating a large number of CNS cancers by methylation..$^{27,28}$ Other medulloblastoma subgrouping methods, such as mRNA sequencing (NanoString, NanoString Technologies, Inc.), have been used with some evidence of benefit to determine medulloblastoma subgroup ${ }^{29}$; however, DNA methylation seems superior. ${ }^{30}$ There is also information suggesting that IHC is not as accurate as DNA methylation, specifically for the identification of WNT-driven medulloblastoma. ${ }^{31}$ Interpretation of certain IHC tests, such as nuclear $\beta$-catenin expression, can be challenging and operator-dependent, and, importantly, nuclear expression of $\beta$-catenin in adult medulloblastoma is not associated with as good a prognosis as it is in children in available retrospective studies. ${ }^{32}$ In addition, many smaller practices may not have the capacity to perform certain IHC analysis (eg, YAP1). Finally, although IHC may yield a quicker diagnosis, not every molecular change will result in a corresponding change at the protein level, potentially increasing false-negative results, as seen in gliomas. ${ }^{33,34}$

\section{Evaluations and Interventions at Baseline and} Throughout the Course of the Disease

Adults with medulloblastoma typically tolerate $\mathrm{CSI}^{35}$ and chemotherapy ${ }^{36}$ worse than children, despite most of them being young adults aged $<40$ years. In addition, as with children, adults with medulloblastoma are at risk for multiple chronic adverse effects from the tumor and therapy, impacting symptom burden, quality of life, and endocrine and neurocognitive function. These treatment sequelae have a potential impact on social outcomes, such as the ability to return to previous activities, including work and education. However, the acute, subacute, and chronic effects of the tumor and therapy have not been studied systematically in adults with medulloblastoma. ${ }^{37,38}$ These patients would benefit from specific evaluations and interventions at baseline and throughout the course of the disease that are well established in pediatric and AYA guidelines ${ }^{39,40}$ but currently are not included in the NCCN Guidelines for Adult Medulloblastoma, such as those related to fertility preservation, parenting, employment attainment and retention, psychosocial support (including participation in support and educational groups), and insurance/financial issues. ${ }^{1}$ The workshop consensus was to recommend inclusion of the following items in the adult medulloblastoma algorithm, at a 
minimum: discuss contraception before initiating therapy; discuss risk of infertility caused by therapy and fertility preservation; offer psychologic support, such as participation in support groups; perform an evaluation for symptoms and medical management (endocrine, vision, hearing, neurocognitive deficits); and offer referral to neurorehabilitation both at initial diagnosis and at any other time as needed throughout the course of the disease. $^{41-43}$

\section{Avoid Delays in Therapy After Initial Diagnosis}

Although there is limited evidence in adults, ${ }^{44}$ it has been shown that delays in starting CSI after diagnosis in children is associated with worse outcome ${ }^{45}$ and therefore most pediatric trials require that CSI begin within 30 days of tumor resection. In the pilot multicenter feasibility trial NOA-07 (ClinicialTrials.gov identifier: NCT01614132), in which adults with newly diagnosed medulloblastoma were treated with CSI and concurrent and adjuvant chemotherapy, the median time from resection to the start of radiochemotherapy was 53 days, and the upcoming EORTC and Alliance trials for newly diagnosed adult medulloblastoma will allow a maximum of 42 days from resection. Even though these relatively short times to treatment initiation can be difficult to accomplish, especially outside of clinical trials, increasing awareness about the importance of avoiding delays in therapy was identified as a critical need by workshop attendees. Shortening the time from resection to initiation of therapy can face multiple barriers and requires several layers of intervention, including patient and healthcare team education, and efforts to expedite referrals between local centers and specialized centers for surgical intervention, molecular testing, CSI, and overall recommendations regarding management. Some of these barriers can be overcome by using telehealth resources to discuss patient management across local and specialized centers.

\section{Incorporate MRI Standards and Criteria \\ for Progression}

The current NCCN Guidelines do not provide specific recommendations for an optimal imaging protocol for evaluating medulloblastoma or criteria for evaluating response to treatment. ${ }^{1}$ As with other primary CNS tumors, MRI with and without contrast is the gold standard for imaging evaluation. However, medulloblastoma has unique imaging characteristics, and findings are far more heterogeneous than those of the most frequent primary CNS tumors in adults (brain metastases, meningioma, glioblastoma). In addition, medulloblastoma has a high propensity for leptomeningeal dissemination with variable radiologic patterns and can be difficult to detect.
The Response Assessment in Pediatric Neuro-Oncology (RAPNO) committee has developed recommendations for response assessment of both adult and pediatric patients with medulloblastoma and other tumors with common leptomeningeal dissemination, which establish imaging standards with minimum sequence acquisition as recommended by the Brain Tumor Imaging Standardization Steering Committee. ${ }^{46,47}$ These standards include performing 3D postcontrast T1-weighted imaging, diffusion-weighted imaging, and postcontrast fluid-attenuated inversion recovery imaging, which were recommended by workshop attendees for inclusion in the NCCN Guidelines. The RAPNO committee also recommends assessment of response using MRI (brain and spine); cerebrospinal fluid cytology; neurologic examination; steroid use; and presence of extra-CNS disease, if applicable. Although these recommendations were primarily designed for use in clinical trials and need prospective validation, proper imaging staging and risk stratification are critical for therapeutic decisions in standard practice, and inadequate imaging practices are linked to worse event-free survival in children. ${ }^{48}$ Furthermore, uniform collection of imaging and evaluation of response assessment was believed to be a beneficial element of registry studies.

\section{Conclusions}

Despite the low incidence of medulloblastoma, a few thousand adults are living with the disease in the United States alone. Guidelines for their optimal care are based on limited high-quality evidence because no randomized trials in adults have been conducted to date. Notwithstanding this paucity of data, adults with medulloblastoma require complex and timely multidisciplinary management by healthcare teams familiarized with their care and needs. These challenges and needs are very similar to those of many other CNS tumors, particularly those with lower incidence. In our opinion, the recommendations outlined in this review are in fact applicable to all CNS tumors.

\section{Acknowledgments}

The authors wish to thank Kathi E. Hanna, PhD, MS, freelance scientific editor, for her assistance with this manuscript.

Submitted June 11, 2020; accepted for publication September 2, 2020.

Disclosures: The authors have disclosed that they have no financial interests, arrangements, affiliations, or commercial interests with the manufacturers of any products discussed in this article or their competitors.

Funding: This work was supported by funding from Cancer Moonshot (M.R.G.).

Correspondence: Marta Penas-Prado, MD, Neuro-Oncology Branch, National Cancer Institute, 9030 Old Georgetown Road, Building 82-213, Bethesda, MD 20892. Email: marta.penas-prado@nih.gov 


\section{References}

1. Nabors LB, Portnow J, Ahluwalia M et al. NCCN Clinical Practice Guidelines in Oncology: Central Nervous System Cancers. Version 2.2020. Accessed May 23, 2020. To view the most recent version, visit NCCN.org

2. Cosman R, Brown CS, DeBraganca KC, et al. Patterns of care in adult medulloblastoma: results of an international online survey. J Neurooncol 2014;120:125-129.

3. Penas-Prado M, Theeler BJ, Cordeiro B, et al. Proceedings of the Comprehensive Oncology Network Evaluating Rare CNS Tumors (NCI-CONNECT) Oligodendroglioma Workshop. Neurooncol Adv 2020;2:vdaa097.

4. Theeler BJ, Dalal Y, Monje M, et al. NCl-CONNECT: Comprehensive Oncology Network Evaluating Rare CNS Tumors-Histone Mutated Midline Glioma Workshop Proceedings. Neurooncol Adv 2020;2:vdaa007.

5. Mansouri A, Zadeh G. NCI-CONNECT rare CNS tumor initiative: current progress and anticipated future impact. Neurooncol Adv 2020;2:vdaa022.

6. Penas-Prado M, Theeler BJ, Cordeiro B, et al. Proceedings of the Comprehensive Oncology Network Evaluating Rare CNS Tumors ( $\mathrm{NCl}-$ CONNECT) Adult Medulloblastoma Workshop [published online August 17, 2020]. Neurooncol Adv, doi.org/10.1093/noajnl/vdaa097

7. Truitt $G$, Gittleman $H$, Leece R, et al. Partnership for defining the impact of 12 selected rare CNS tumors: a report from the CBTRUS and the $\mathrm{NCl}-$ CONNECT. J Neurooncol 2019;144:53-63.

8. Majd N, Mastall M, Hess K, et al. RARE-47. Characterization of adult medulloblastoma: a retrospective review of 200 patients at MD Anderson Cancer Center [abstract]. Neuro Oncol 2019;21(Suppl 6):vi231-232.

9. Majd N, Penas-Prado M. Updates on management of adult medulloblastoma. Curr Treat Options Oncol 2019;20:64.

10. Franceschi $E$, Hofer $S$, Brandes AA, et al. EANO-EURACAN clinical practice guideline for diagnosis, treatment, and follow-up of post-pubertal and adult patients with medulloblastoma. Lancet Oncol 2019;20:e715-728.

11. Penas-Prado M, Armstrong TS, Gilbert MR. Progress in rare central nervous system tumors. Curr Opin Neurol 2019;32:895-906.

12. Elrod JK, Fortenberry JL Jr. Centers of excellence in healthcare institutions: what they are and how to assemble them. BMC Health Serv Res 2017;17(Suppl 1):425.

13. Zhu P, Du XL, Zhu JJ, et al. Improved survival of glioblastoma patients treated at academic and high-volume facilities: a hospital-based study from the National Cancer Database. J Neurosurg 2020:132:491-502.

14. Raj R, Seppä K, Luostarinen T, et al. Disparities in glioblastoma survival by case volume: a nationwide observational study. J Neurooncol 2020;147:361-370.

15. Zhu P, Du XL, Blanco Al, et al. Impact of facility type and volume in low-grade glioma outcomes [published online September 27, 2019]. J Neurosurg, doi: 10.3171/2019.6.JNS19409 https://doi.org/10.3171/2019.6.JNS19409

16. Hauser A, Dutta SW, Showalter TN, et al. Impact of academic facility type and volume on post-surgical outcomes following diagnosis of glioblastoma. J Clin Neurosci 2018;47:103-110.

17. Haque W, Verma $V$, Butler EB, et al. Definitive chemoradiation at high volume facilities is associated with improved survival in glioblastoma. J Neurooncol 2017;135:173-181.

18. Patil VM, Pande N, Chandrasekharan A, et al. Shadow study: randomized comparison of clinic with video follow-up in glioma undergoing adjuvant temozolomide therapy. CNS Oncol 2018;7:CNS14.

19. Reider-Demer M, Raja P, Martin N, et al. Prospective and retrospective study of videoconference telemedicine follow-up after elective neurosurgery: results of a pilot program. Neurosurg Rev 2018;41:497-501.

20. Abu Arja MH, Stanek JR, Morales La Madrid AE, et al. The Latin American Brain Tumor Board teleconference: results of a web-based survey to evaluate participant experience utilizing this resource. Childs Nerv Syst 2019;35:257-265.

21. Qaddoumi I, Mansour A, Musharbash A, et al. Impact of telemedicine on pediatric neuro-oncology in a developing country: the JordanianCanadian experience. Pediatr Blood Cancer 2007;48:39-43.

22. Lee $\mathrm{EQ}$, Weller $\mathrm{M}$, Sul J, et al. Optimizing eligibility criteria and clinical trial conduct to enhance clinical trial participation for primary brain tumor patients. Neuro Oncol 2020;22:601-612.

23. Friedrich $\mathrm{C}$, von Bueren $\mathrm{AO}$, von $\mathrm{Hoff} \mathrm{K}$, et al. Treatment of adult nonmetastatic medulloblastoma patients according to the paediatric HIT 2000 protocol: a prospective observational multicentre study. Eur J Cancer 2013;49:893-903.

24. von Bueren AO, Friedrich $\mathrm{C}$, von Hoff $\mathrm{K}$, et al. Metastatic medulloblastoma in adults: outcome of patients treated according to the HIT2000 protocol. Eur J Cancer 2015;51:2434-2443.

25. Louis DN, Perry A, Reifenberger G, et al. The 2016 World Health Organization Classification of Tumors of the Central Nervous System: a summary. Acta Neuropathol 2016;131:803-820.
26. Capper D, Jones DTW, Sill M, et al. DNA methylation-based classification of central nervous system tumours. Nature 2018;555:469-474.

27. Siegel C, Armstrong T, Chung HJ, et al. PATH-40. Standardized molecular analysis of primary central nervous system (CNS) tumors in a research based clinical practice. An introduction to the CNS tumor next generation sequencing (NGS) panel. Neuro Oncol 2017;19(Suppl 6):vi179-180.

28. Capper D, Stichel D, Sahm F, et al. Practical implementation of DNA methylation and copy-number-based CNS tumor diagnostics: the Heidelberg experience. Acta Neuropathol 2018;136:181-210.

29. D'Arcy CE, Nobre LF, Arnaldo A, et al. Immunohistochemical and nanoString-based subgrouping of clinical medulloblastoma samples. J Neuropathol Exp Neurol 2020;79:437-447.

30. Korshunov A, Chavez L, Northcott PA, et al. DNA-methylation profiling discloses significant advantages over NanoString method for molecular classification of medulloblastoma. Acta Neuropathol 2017;134:965-967.

31. Pietsch $T$, Schmidt R, Remke M, et al. Prognostic significance of clinical, histopathological, and molecular characteristics of medulloblastomas in the prospective HIT2000 multicenter clinical trial cohort. Acta Neuropathol 2014;128:137-149.

32. Korshunov A, Remke M, Werft W, et al. Adult and pediatric medulloblastomas are genetically distinct and require different algorithms for molecular risk stratification. J Clin Oncol 2010;28:3054-3060.

33. Gondim DD, Gener MA, Curless KL, et al. Determining IDH-mutational status in gliomas using IDH1-R132H antibody and polymerase chain reaction. Appl Immunohistochem Mol Morphol 2019;27:722-725.

34. Roshandel AK, Busch CM, Mullekom JV, et al. The predictive capability of immunohistochemistry and DNA sequencing for determining TP53 functional mutation status: a comparative study of 41 glioblastoma patients. Oncotarget 2019;10:6204-6218.

35. Brown AP, Barney CL, Grosshans DR, et al. Proton beam craniospinal irradiation reduces acute toxicity for adults with medulloblastoma. Int $\mathrm{J}$ Radiat Oncol Biol Phys 2013;86:277-284

36. Beier $D$, Proescholdt $M$, Reinert $C$, et al. Multicenter pilot study of radiochemotherapy as first-line treatment for adults with medulloblastoma (NOA-07). Neuro Oncol 2018;20:400-410.

37. Harrison RA, Kesler SR, Johnson JM, et al. Neurocognitive dysfunction in adult cerebellar medulloblastoma. Psychooncology 2019;28:131-138.

38. Dirven L, Luerding R, Beier $D$, et al. Neurocognitive functioning and health-related quality of life in adult medulloblastoma patients: long-term outcomes of the NOA-07 study. J Neurooncol 2020;148:117-130.

39. Landier W, Bhatia S, Eshelman DA, et al. Development of risk-based guidelines for pediatric cancer survivors: the Children's Oncology Group Long-Term Follow-Up Guidelines from the Children's Oncology Group Late Effects Committee and Nursing Discipline. J Clin Oncol 2004;22:4979-4990.

40. Coccia PF, Papo AS, Beaupin L, et al. NCCN Clinical Practice Guidelines in Oncology: Adolescent and Young Adult (AYA) Oncology. Version 2.2018. Accessed May 23, 2020. To view the most recent version, visit NCCN.org

41. Amidei C. Symptom-based interventions to promote quality survivorship. Neuro Oncol 2018;20(Suppl 7):vii27-39.

42. Leeper $\mathrm{H}$, Milbury K. Survivorship care planning and implementation in neuro-oncology. Neuro Oncol 2018;20(Suppl 7):vii40-46.

43. Leeper HE, Acquaye AA, Bell S, et al. Survivorship care planning in neurooncology. Neurooncol Pract 2018;5:3-9.

44. Abacioglu U, Uzel O, Sengoz M, et al. Medulloblastoma in adults: treatment results and prognostic factors. Int J Radiat Oncol Biol Phys 2002;54:855-860

45. Taylor RE, Bailey CC, Robinson K, et al. Results of a randomized study of preradiation chemotherapy versus radiotherapy alone for nonmetastatic medulloblastoma: the International Society of Paediatric Oncology/United Kingdom Children's Cancer Study Group PNET-3 Study. J Clin Oncol 2003;21:1581-1591.

46. Warren KE, Vezina G, Poussaint TY, et al. Response assessment in medulloblastoma and leptomeningeal seeding tumors: recommendations from the Response Assessment in Pediatric Neuro-Oncology committee. Neuro Oncol 2018;20:13-23.

47. Ellingson BM, Bendszus M, Boxerman J, et al. Consensus recommendations for a standardized brain tumor imaging protocol in clinical trials. Neuro Oncol 2015;17:1188-1198.

48. Packer RJ, Gajjar A, Vezina G, et al. Phase III study of craniospinal radiation therapy followed by adjuvant chemotherapy for newly diagnosed average-risk medulloblastoma. J Clin Oncol 2006;24:4202-4208. 